Article

\title{
On the Relationship of Cryptocurrency Price with US Stock and Gold Price Using Copula Models
}

\author{
Jong-Min Kim ${ }^{1}\left(\mathbb{D}\right.$, Seong-Tae $\mathrm{Kim}^{2}{ }^{\mathbb{D}}$ and Sangjin $\mathrm{Kim}^{3, *}{ }^{3}$ \\ 1 Statistics Discipline, University of Minnesota at Morris, Morris, MN 56267, USA; jongmink@morris.umn.edu \\ 2 Department of Mathematics, North Carolina A\&T State University, Greensboro, NC 27411, USA; \\ skim@ncat.edu \\ 3 Department of Management and Information Systems, Dong-A University, Busan 49236, Korea \\ * Correspondence: skim10@dau.ac.kr
}

Received: 15 September 2020; Accepted: 20 October 2020; Published: 23 October 2020

\begin{abstract}
This paper examines the relationship of the leading financial assets, Bitcoin, Gold, and S\&P 500 with GARCH-Dynamic Conditional Correlation (DCC), Nonlinear Asymmetric GARCH DCC (NA-DCC), Gaussian copula-based GARCH-DCC (GC-DCC), and Gaussian copula-based Nonlinear Asymmetric-DCC (GCNA-DCC). Under the high volatility financial situation such as the COVID-19 pandemic occurrence, there exist a computation difficulty to use the traditional DCC method to the selected cryptocurrencies. To solve this limitation, GC-DCC and GCNA-DCC are applied to investigate the time-varying relationship among Bitcoin, Gold, and S\&P 500. In terms of log-likelihood, we show that GC-DCC and GCNA-DCC are better models than DCC and NA-DCC to show relationship of Bitcoin with Gold and S\&P 500. We also consider the relationships among time-varying conditional correlation with Bitcoin volatility, and S\&P 500 volatility by a Gaussian Copula Marginal Regression (GCMR) model. The empirical findings show that S\&P 500 and Gold price are statistically significant to Bitcoin in terms of log-return and volatility.
\end{abstract}

Keywords: cryptocurrency; gold; S\&P 500; GARCH; DCC; copula

\section{Introduction}

Knowing the relationships of the cryptocurrency market with either the US stock market or commodity market will be very useful to manage investors' portfolios and how many portions of their investment money will be allocated to cryptocurrency for their secure and profitable investment plan. Cryptocurrency is a digital or virtual currency that is exchanged between peers without the need for a third party [1]. The key features of the cryptocurrency include that there is no central system to manage the transactions of cryptocurrencies, and they are classified as a commodity by the U.S. Commodity Futures Trading Commission (CFTC). The first cryptocurrency, Bitcoin, operates with block-chain technology, in which a secure system of accounting is used that transfers ownership. The cryptocurrency market is an attractive emerging market for investment, but this market revealed downfalls such as cryptocurrency hacking news. For example, in May 2019, hackers stole \$40 million worth of Bitcoin from Binance, one of the largest cryptocurrency exchanges in the world. Therefore, investors themselves have to take a high risk from cryptocurrency investment. However, the recent cryptocurrency market is a bull market where the Bitcoin price is equal to the USD 10,806.90 as of 30 September 2020, but the Bitcoin price has severely fluctuated since the maximum Bitcoin price at the USD 19,783.06 on 17 December 2017. Despite a series of negative events in this market, investing cryptocurrency is gaining popularity among investors to make their own money. Consequently, economic entities are interested in the dynamic relationships among the cryptocurrency market, commodity market, and stock market. 
There have been many studies on the analysis of the exchange rates of cryptocurrency [2]. Recently, Hyun et al. [3] examined dependence relationships among the five well-known cryptocurrencies (Bitcoin, Ethereum, Litecoin, Ripple, and Stella) using a copula directional dependence. Kim et al. [4] studied the volatility of nine well-known cryptocurrencies-Bitcoin, XRP, Ethereum, Bitcoin Cash, Stella, Litecoin, TRON, Cardano, and IOTA using several GARCH models and Bayesian Stochastic Volatility (SV) models. Klein et al. [5] employed the BEKK [6] GARCH model to estimate time-varying conditional correlations between gold and Bitcoin. In terms of portfolio management, Aslanidisa, Barivierab and Martínez-Ibañeza [7] considered Dynamic Conditional Correlation (DCC) with daily price data (21 May 2014, to 27 September 2018), pairs of four cryptocurrencies (Bitcoin, Dash, Monero, and Ripple), and three traditional financial assets (Standard \& Poors 500 Composite (SP500), S\&P US Treasury bond 7-10Y index (BOND), and Gold Bullion LBM) [8-11]. Guesmi et al. [12] examined the dynamics of Bitcoin and other financial assets using the VARMA $(1,1)$-DCC-GJR-GARCH model and found that Bitcoin provides diversification and hedging opportunities for investment. Hyun et al. [3] already applied the copula approach to cryptocurrency because no assumption is needed such as normality, linearity, and independence of the errors from the proposed model.

In this study, we aim to apply the copula-based GARCH-DCC models $[3,13,14]$ to see the recent time varying correlations between the cryptocurrency market and US stock price or between the cryptocurrency market and commodity market price after the slump of the cryptocurrency market price since 2018. The copula-based GARCH-DCC models are compared to the GARCH-DCC models in the empirical data analysis $[8,15-17]$ which shows that copula-based GARCH-DCC models has better model than GARCH-DCC models. A copula is a multivariate distribution function described on the unit $[0,1]^{n}$ with uniformly distributed marginal [18]. Our result also leaded to the same conclusion as the previous researches. Furthermore, because of the failure of the ordinary least regression to capture the heteroscedasticity with high volatility financial data, we use the Gaussian Copula Marginal Regression (GCMR) models [19] which can consider the heteroscedasticity and non-normality of the financial data to test our alternative hypothesis that Bitcoin is statistically significant by log-returns of S\&P 500 and Gold price in terms of log-return. We also test the current volatility of log-returns of Bitcoin can be statistically significant with the current and lagged volatilities of the other assets (S\&P 500 and Gold price). We also test that the time varying correlations of log-returns of Bitcoin and S\&P 500 can be statistically significant with the current volatilities of the Bitcoin and S\&P 500 .

The paper is organized as follows. Section 2 reviews econometric methodologies that will be used in this paper. Section 3 describes data and discusses empirical data analysis. Section 4 provides the conclusion and our related future study.

\section{Econometrical Methods}

This section introduces the volatility model, dynamic correlation coefficient, copula, and their combinations. The description of econometric models is not comprehensive but selective to understand the dynamic relationships among the three markets.

\subsection{GARCH Models}

Let $S_{t}$ be a price time series at time $t$. For a $\log$ return series $r_{t}=\log \left(\frac{S_{t}}{S_{t-1}}\right)$, we let $a_{t}=r_{t}-E_{t-1}\left[r_{t}\right]$ be the innovation at time $t$. Then $a_{t}$ follows a $\operatorname{GARCH}(p, q)$ model if $a_{t}=h_{t} \epsilon_{t}$

$$
h_{t}^{2}=\alpha_{0}+\sum_{i=1}^{q} \alpha_{i} a_{t-i}^{2}+\sum_{j=1}^{p} \beta_{j} h_{t-j}^{2}
$$

where $\left\{\epsilon_{t}\right\}$ is a sequence of independent and identically distributed random variables with mean 0 and variance $1, \alpha_{0}>0, \alpha_{i} \geq 0, \beta_{j} \geq 0$, and $\sum_{i=1}^{\max (p, q)}\left(\alpha_{i}+\beta_{j}\right) \leq 1$. All members of the family of GARCH models can be obtained from a transformation of the conditional standard deviation, $h_{t}$, determined by the transformation of the innovations, $a_{t}$, and lagged transformed conditional standard 
deviations. An extensive discussion on the nested GARCH models is given in Hentschel [20]. Since the conditional variance in the GARCH model did not properly respond to positive and negative shocks, Engel and $\mathrm{Ng}$ [21] also proposed one of the popular nonlinear asymmetric GARCH (NAGARCH) models as follows:

$$
h_{t}^{2}=\alpha_{0}+\sum_{i=1}^{q} \alpha_{i}\left(a_{t-i}-\gamma_{i} h_{t-i}\right)^{2}+\sum_{j=1}^{q} \beta_{j} h_{t-j^{\prime}}^{2}
$$

where $\alpha_{0}>0, \alpha_{i} \geq 0, \beta_{j} \geq 0$ for $i=1,2, \ldots, p$ and $j=1,2, \ldots, q$. In the model, the distance $\gamma_{i} h_{t-i}$ moves the news impact curve to the right, and the parameter $\gamma_{i}$ of stock returns is estimated to be positive. It indicates that negative returns increase future volatility with larger amounts than positive returns of the same magnitude.

The T-GARCH model, which can capture the asymmetric effect in the volatility is given by

$$
h_{t}^{2}=\alpha_{0}+\sum_{i=1}^{q} \alpha_{i}\left(\left|a_{t-i}\right|-\eta_{i} a_{t-i}\right)^{2}+\sum_{j=1}^{p} \beta_{j} h_{t-j^{\prime}}^{2}
$$

where the asymmetric parameter $\eta$ satisfies the condition $-1<\eta<1$. For the model selection of the GARCH $(1,1)$ models considered, we use the Akaike Information Criterion (AIC). Besides, this study also considers the Student-t errors to take into account the possible fatness of the distribution tails of $a_{t}$.

\subsection{DCC and Copula DCC Models}

To investigate the time-varying correlations among multivariate returns, we adopt the DCC model, which incorporates the flexibility of univariate GARCH models and the harmonicity of correlation estimation functions. In the DCC model in [6,22], the correlation matrix is time-varying, and the covariance matrix can be decomposed into:

$$
H_{t}=D_{t} R D_{t}=\rho_{i j} \sqrt{h_{i j, t} h_{i j, t}}, \text { where } D_{t}=\operatorname{diag}\left(\sqrt{h_{11, t}}, \ldots, \sqrt{h_{n n, t}}\right)
$$

containing the time-varying standard deviations is obtained from GARCH models, and $R$ is the constant conditional correlation (CCC) proposed by Bollerslev [23], which is defined as $R=T^{-1} \sum_{i=t}^{T} v_{t} v_{t}^{\prime}$, where $v_{t}=\frac{r_{t}-\mu}{\sigma_{t}}$, and $\mu$ is a vector of expected returns. The DDC in [24] is a time-varying extension of the CCC, which has the following structure:

$$
R_{t}=\operatorname{diag}\left(Q_{t}\right)^{-\frac{1}{2}} Q_{t} \operatorname{diag}\left(Q_{t}\right)^{-\frac{1}{2}}
$$

where $Q_{t}=R+\alpha\left(v_{t-1} v_{t-1}^{\prime}-R\right)+\beta\left(Q_{t-1}-R\right)$.

Note that to ensure stationarity, nonnegative $\alpha$ and $\beta$ satisfy the constraint $\alpha+\beta<1$, and $Q_{t}$ is positive definite which makes $R_{t}$ positive definite. Off-diagonal elements in the covariance matrix $Q_{t}$ are the correlation coefficients between pairwise indexes among Bitcoin, Gold, and S\&P 500 at time $t$. In this paper, we use the "dcc.estimation" function in the "ccgarch" on R package [24,25] to estimate each conditional correlation.

We consider another statistical approach to address the correlation among multivariate time series. Sklar [26] suggested copular functions to build joint multivariate distributions. The copula models we consider here are Gaussian copulas which are used to estimate the time-varying correlation matrix of the DCC model. A copula is an efficient way to characterize and model correlated multivariate random variables. Therefore, we consider the time-varying conditional correlation in the copula 
framework. Let a random vector $\left(X_{1}, \ldots, X_{p}\right)$ have marginal distribution functions $F_{i}\left(x_{i}\right)=P\left(X_{i} \leq x_{i}\right)$ for $i=1, \ldots, p$. The dependence function, $C$, for all $u_{1}, \ldots, u_{n} \in[0,1]^{n}$ can be defined as:

$$
\begin{gathered}
C\left(u_{1}, \ldots, u_{n}\right)=P\left(F_{1}\left(X_{1}\right) \leq u_{1}, \ldots, F_{n}\left(X_{n}\right) \leq u_{n}\right) \\
C\left(u_{1}, \ldots, u_{n}\right)=F\left(F_{1}^{-1}\left(u_{1}\right), \ldots, F_{1}^{-1}\left(u_{n}\right)\right) .
\end{gathered}
$$

In this study, we estimate $\operatorname{DCC}\left(\hat{\rho}_{t}\right)$ by a Gaussian copula function whose conditional density is defined as:

$$
c_{t}\left(u_{1 t}, \ldots, u_{n t} \mid R_{t}\right)=\frac{f_{t}\left(F_{1}^{-1}\left(u_{1 t}\right), \ldots, F_{1}^{-1}\left(u_{n t}\right) \mid R_{t}\right)}{\prod_{i=1}^{n} f_{i}\left(F_{1}^{-1}\left(u_{i t}\right)\right)},
$$

where $R_{t}$ is the correlation matrix implied by the covariance matrix, $u_{i t}=F_{i t}\left(r_{i t} \mid \mu_{i t}, h_{i t}, v_{t}, \tau_{i}\right)$ is the probability integral transformed values estimated by the GARCH process, and $F_{1}^{-1}\left(u_{i t} \mid \tau\right)$ represents the quantile transformation. We estimate each conditional correlation via the "cgarchspec" function in the $\mathrm{R}$ package "rmgarch" implementing the Gaussian copula [27,28]. In particular, our model applies the Gaussian copula to estimate the conditional covariance matrix. We propose four different DCC-related models: the GARCH-DCC (DCC) model, Nonlinear Asymmetric-GARCH-DCC (NA-DCC) model, Gaussian copula-based GARCH-DCC (GC-DCC) model, and Gaussian copula-based nonlinear asymmetric GARCH-DCC (GCNA-DCC) model to see the dynamic conditional correlations between Bitcoin and S\&P 500 and between Bitcoin and Gold.

\subsection{Gaussian Copula Marginal Regression (GCMR) Model}

Gaussian Copula Marginal Regression (GCMR) is another methodology used in this study to capture the relationship, where dependence is expressed in the correlation matrix of a multivariate Gaussian distribution $[19,29]$. Let $F\left(\cdot \mid x_{i}\right)$ be a marginal cumulative distribution depending on a vector of covariates $x_{i}$. If a set of $\mathrm{n}$ dependent variables in $Y_{i}$ is considered, then the joint cumulative distribution function is in the Gaussian copula regression defined by

$$
\operatorname{Pr}\left(Y_{1} \leq y_{1}, \ldots, Y_{1} \leq y_{1}\right)=\Phi_{n}\left\{\varepsilon_{1}, \ldots, \varepsilon_{n} ; P\right\},
$$

where $\varepsilon_{i}=\Phi^{-1}\left\{F\left(y_{i} \mid x_{i}\right)\right\} . \Phi(\cdot)$ and $\Phi_{n}(\cdot ; P)$ indicate the univariate and multivariate standard normal cumulative distribution functions, respectively. $P$ denotes the correlation matrix of the Gaussian copula. Masarotto and Varin [19] propose an equivalent formulation of the Gaussian copula model linking each variable $Y_{i}$ to a vector of covariates $x_{i}$ as follows:

$$
Y_{i}=h\left(x_{i}, \varepsilon_{i}\right),
$$

where $\varepsilon_{i}$ indicates a stochastic error. In particular, the Gaussian copula regression model assumes that $h\left(x_{i}, \varepsilon_{i}\right)=F^{-1}\left\{\Phi\left(\varepsilon_{i}\right) \mid x_{i}\right\}$ and $\varepsilon$ has a multivariate standard normal distribution with correlation matrix $P$. The advantages of using GCMR are to keep the marginal univariate distributions for each variable and to have multivariate normal errors for the joint distribution.

\section{Empirical Analysis and Results}

In this section, we apply the proposed methods to the three selected price time series. Given the sensitivity of the periods in predicting the volatility of financial time-series return data such as cryptocurrencies, we examine two different periods, more recent and short- and long-term periods. The sample consists of the daily log-returns of the nine cryptocurrencies over the period from 2 January 2018 to 21 September 2020. The log-returns of Bitcoin (BTC) and S\&P 500 are denoted by LBTC and LSP, respectively. We obtained our Bitcoin data from a financial website [30], Gold data from Prof. Werner Antweiler's website [31] at the University of British Columbia Sauder School of Business, and S\&P 500 data from the Yahoo finance website [32]. 
Figure 1 compares the pattern of prices of Bitcoin, Gold, and S\&P 500 at the original scale since January 2018. The graphs appear to have a significant pairwise positive relationship after the COVID-19 pandemic occurrence. Therefore, with the log-returns of prices of Bitcoin, Gold, and S\&P 500 (LBTC, LGD, LSP), we test if there is the significant pairwise correlation among LBTC, LGD, and LSP in this period using three correlations measures, the Pearson correlation method with the linear relationship assumption and Spearman and Kendall rank correlations as non-parametric methods. The data provided no statistically significant pairwise relationships among the three variables of prices as seen in Table 1. We also summarized descriptive statistics of the log return data of the cryptocurrencies such as mean, skewness, and kurtosis as well as the five-number summary statistics in Table 2. In Table 2, it is recognized that the standard deviation of LBTC is larger than those of LGD and LSP, which means that LBTC has a higher risk than LGD and LSP in terms of investment. Besides, the value of kurtosis in LBTC is greater than 3, meaning heavy tails while LGD and LSP have values less than 3, meaning light tails compared to a normal distribution. The LBTC and LSP are left-skewed while LGD is right-skewed. It means that the prices of Bitcoin and the S\&P 500 will more likely be decreased soon, but the price of Gold will more likely be increased shortly.
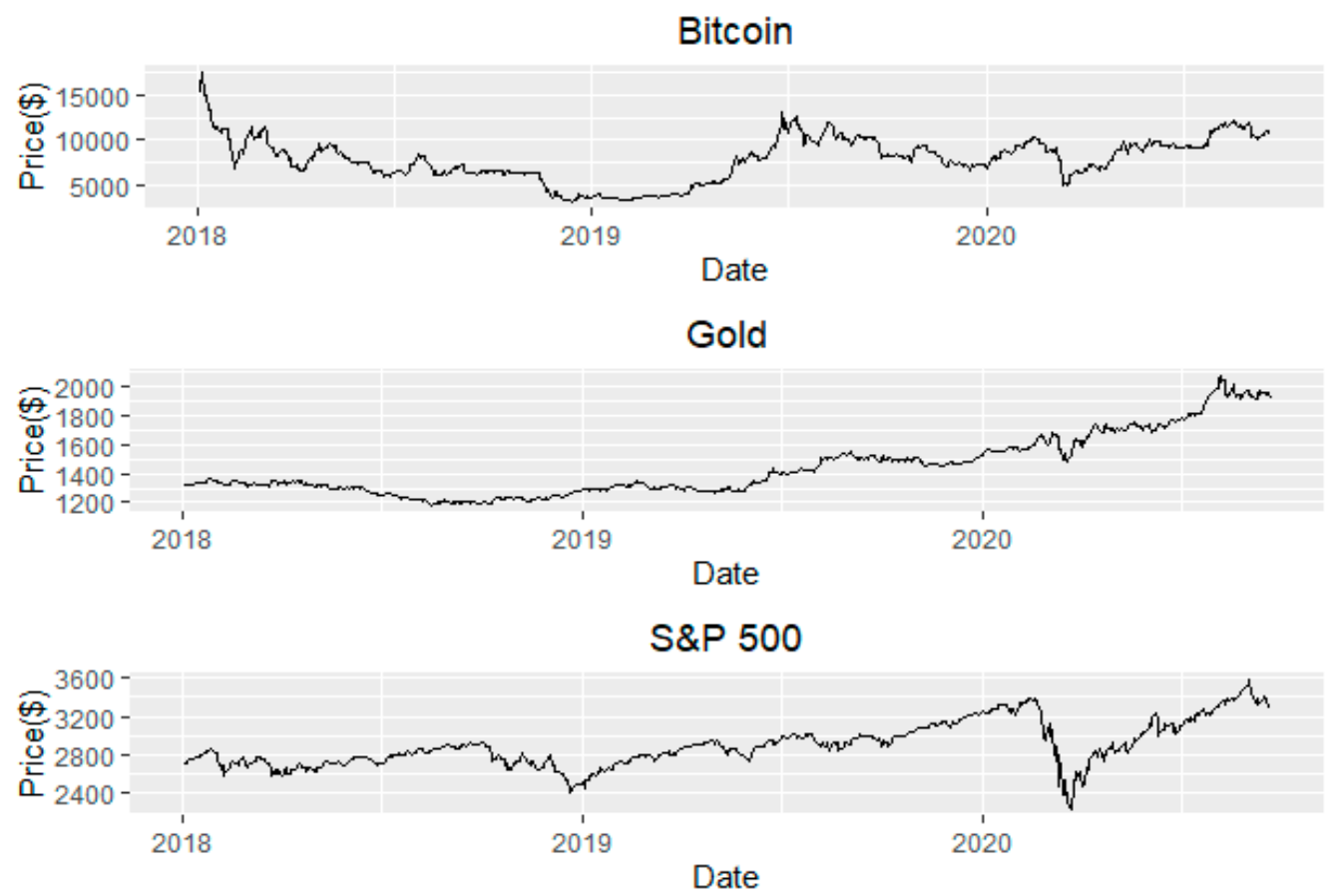

Figure 1. Prices of Bitcoin, Gold, and S\&P 500.

Table 1. Correlation coefficients of log-return of Bitcoin (LBTC), log-return of Gold (LGD), and log-returns of S\&P 500 (LSP) with Pearson, Spearman, and Kendall.

\begin{tabular}{ccccc}
\hline \multirow{3}{*}{ Pearson } & & LBTC & LGD & LSP \\
\hline \multirow{3}{*}{ Spearman } & LBTC & 1 & 0.202 & 0.240 \\
& LGD & 0.202 & 1 & 0.255 \\
& LSP & 0.240 & 0.255 & 1 \\
\hline \multirow{3}{*}{ Kendall } & LBTC & 1 & 0.134 & 0.095 \\
& LGD & 0.134 & 1 & 0.084 \\
& LSP & 0.095 & 0.084 & 1 \\
\hline & LBTC & 1 & 0.090 & 0.064 \\
& LGD & 0.090 & 1 & 0.060 \\
\hline
\end{tabular}


Table 2. Summary statistics of the log-return of Bitcoin (LBTC), log-return of Gold (LGD), and log-returns of S\&P 500 (LSP).

\begin{tabular}{cccc}
\hline & LBTC & LGD & LSP \\
\hline Min & -0.465 & -0.053 & -0.128 \\
Q1 & -0.016 & -0.004 & -0.004 \\
Q2 & 0.001 & 0.0004 & 0.001 \\
Mean & -0.0005 & 0.0006 & 0.0003 \\
Q3 & 0.019 & 0.005 & 0.007 \\
Max & 0.203 & 0.051 & 0.090 \\
SD & 0.049 & 0.009 & 0.015 \\
Skewness & -1.579 & -0.268 & -1.067 \\
Kurtosis & 17.662 & 9.311 & 18.960 \\
\hline
\end{tabular}

Since a causality between two variables may exist although there is no correlation as in Table 1 , we tested if there is linear Granger causality with each lag of 1,2, and 3 using the "grangertest" function in the "lmtest" R package [33]. That is, we consider the causality from LBTC to LSP and vice versa and from LBTC to LGD and vice versa. Table 3 shows the results of linear Granger causality tests at lag 1, 2 , and 3, respectively. As seen in Table 3, there is no statistically significant causality among LBTC, LSP and LGD at the lag 1 but there is statistically significant causality among (LBTC, LSP) and (LBTC, LGD) at the lag 2 and the lag 3.

Table 3. The result of linear Granger causality with lag of 1,2, and 3. There is no Granger causality between the log-returns of Bitcoin (LBTC) and S\&P 500 (LSP) and Bitcoin (LBTC) and Gold (LGD), respectively.

\begin{tabular}{ccccccc}
\hline & \multicolumn{2}{c}{ Lag 1 } & \multicolumn{2}{c}{ Lag 2 } & \multicolumn{2}{c}{ Lag 3 } \\
\hline Causality & F-stat & $p$-val & F-stat & $p$-val & F-stat & $p$-val \\
Bitcoin $\rightarrow$ S\&P 500 & 2.656 & 0.104 & 8.153 & 0.000 & 5.520 & 0.001 \\
S\&P 500 $\rightarrow$ Bitcoin & 0.530 & 0.467 & 0.120 & 0.887 & 0.257 & 0.857 \\
Bitcoin $\rightarrow$ Gold & 0.034 & 0.854 & 4.217 & 0.015 & 3.788 & 0.010 \\
Gold $\rightarrow$ Bitcoin & 0.868 & 0.352 & 1.882 & 0.153 & 2.413 & 0.066 \\
\hline
\end{tabular}

Figure 2 shows the volatilities of log-returns of Bitcoin, Gold, and S\&P 500 with the models of GARCH and NAGARCH. The GARCH volatilities are larger than those of the NAGARCH, while the pattern of volatility is similar between the two models. In each of the two plots, the level of volatilities (or risk) among the log-returns of Bitcoin, Gold, and S\&P 500 is in the order of Bitcoin, S\&P 500, and Gold.

To investigate the volatilities of the LBTC, LGD, LSP, we consider three different GARCH models which include two asymmetric GARCH models, T-GARCH $(1,1)$, and Nonlinear Asymmetric-GARCH $(1,1)$, and one standard-GARCH $(1,1)$. Table 4 reports the result of log-likelihood to choose an optimal model among the three models. The standard-GARCH $(1,1)$ model achieved the minimum AIC scores meaning a better fit across LBTC, LSP, and LGD. 
Daily GARCH Volatility
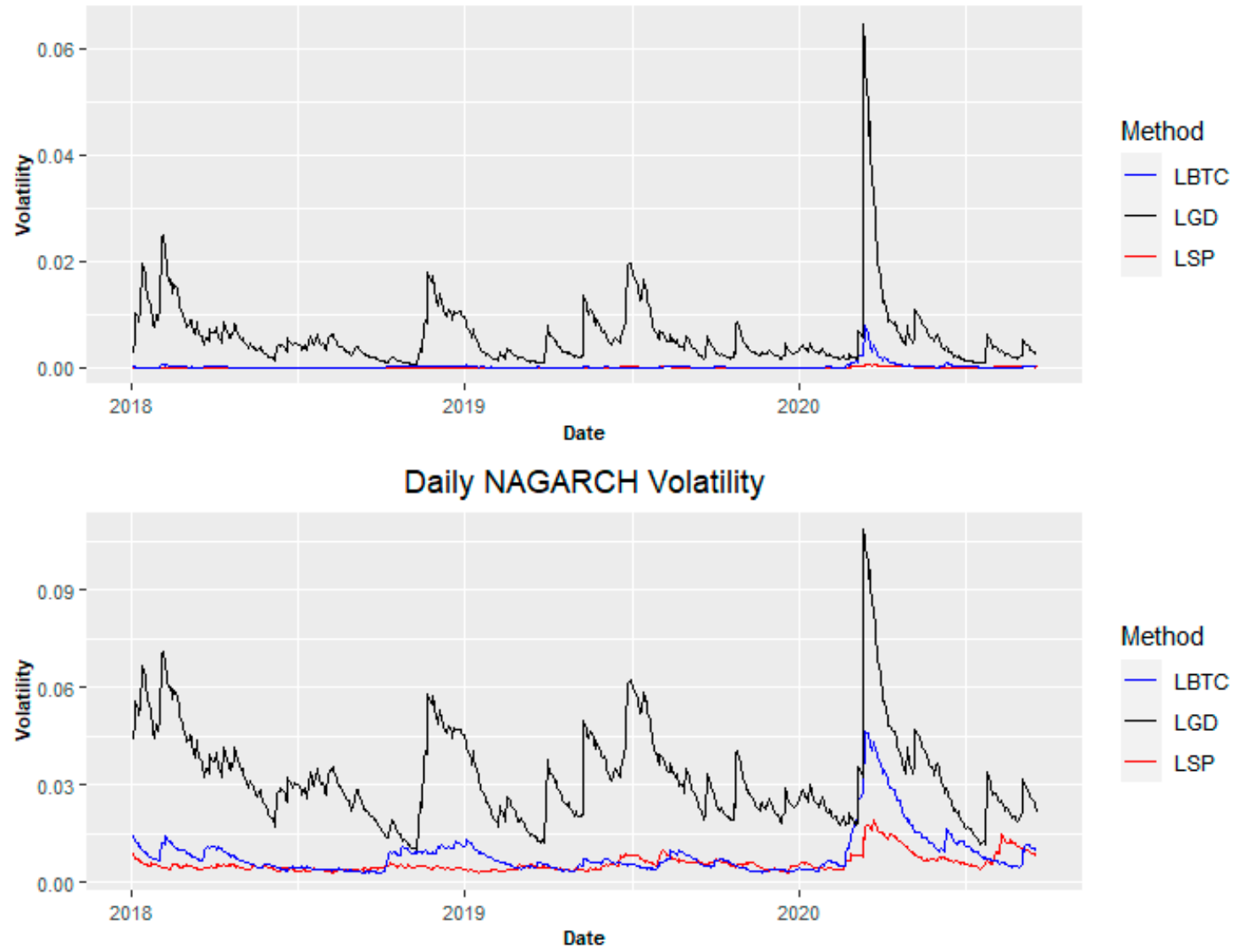

Figure 2. Daily volatility plots for LBTC, LSP, LGD with GARCH $(1,1)$, and Nonlinear Asymmetric-GARCH $(1,1)$.

Table 4. The result of Akaike Information Criterion (AIC) scores to select the best model among three different GARCH models. The Standard-GARCH $(1,1)$ model has maximum values of log-likelihood (LH) from the log-return of Bitcoin (LBTC), log-return of Gold (LGD), and log-returns of S\&P 500 (LSP). A higher LH indicates a better fit.

\begin{tabular}{cccc}
\hline & LBTC & LSP & LGD \\
\hline T-GARCH $(1,1)$ & 1568.405 & 1702.325 & 797.6006 \\
NA-GARCH $(1,1)$ & 2014.815 & 2139.722 & 1163.852 \\
Standard-GARCH $(1,1)$ & 2106.239 & 2246.397 & 1206.569 \\
\hline
\end{tabular}

We apply a standard-GARCH $(1,1)$ model to LBTC, LSP, and LGD to check if there exists volatility clustering. Table 5 shows the results of the model fits based on the standard-GARCH $(1,1)$ model. The coefficient $\beta_{1}$ is the effect of the conditional variance at time $t-1$ on the conditional variance at time $t$, so a high value close to one indicates a longer persistency of the volatility shock. Hence, the estimates of $\beta_{1}^{\prime} s$ in the table explain the amount of volatility clustering. Likewise, there exist consistent volatility clusterings throughout all models since all $p$ values of $\beta_{1}$ s are closed to 0 at $\alpha=0.05$. 
Table 5. The results of the standard-GARCH $(1,1)$ model with the log-return of Bitcoin (LBTC), log-return of Gold (LGD), and log-returns of S\&P 500 (LSP) where $\alpha_{0}, \alpha_{1}$, and $\beta_{1}$ are from Equation (1).

\begin{tabular}{|c|c|c|c|c|}
\hline \multicolumn{5}{|c|}{ Standard-GARCH Model Fit with LBTC } \\
\hline & Estimate & S.E & $t$-Value & $p$-Value \\
\hline$\alpha_{0}$ & 0.000 & 0.000 & 2.700 & 0.007 \\
\hline$\alpha_{1}$ & 0.244 & 0.051 & 4.794 & 0.000 \\
\hline$\beta_{1}$ & 0.755 & 0.040 & 18.707 & 0.000 \\
\hline$t$-distribution parameter & 5.547 & 1.167 & 4.752 & 0.000 \\
\hline \multicolumn{5}{|c|}{ Standard-GARCH Model Fit with LSP } \\
\hline$\alpha_{0}$ & 0.000 & 0.000 & 2.026 & 0.043 \\
\hline$\alpha_{1}$ & 0.097 & 0.029 & 3.319 & 0.000 \\
\hline$\beta_{1}$ & 0.867 & 0.039 & 22.494 & 0.000 \\
\hline$t$-distribution parameter & 6.260 & 1.522 & 4.111 & 0.000 \\
\hline \multicolumn{5}{|c|}{ Standard-GARCH Model Fit with LGD } \\
\hline$\alpha_{0}$ & 0.000 & 0.000 & 0.964 & 0.335 \\
\hline$\alpha_{1}$ & 0.277 & 0.148 & 1.560 & 0.084 \\
\hline$\beta_{1}$ & 0.878 & 0.033 & 32.097 & 0.000 \\
\hline$t$-distribution parameter & 2.315 & 0.337 & 10.516 & 0.000 \\
\hline
\end{tabular}

Furthermore, we checked the normality of the data and determined if a good fit had been achieved based on the Ljung-Box test which is a classical hypothesis test whose null hypothesis is that the autocorrelations between the population series values are zero. Table 6 shows the results of the Jarque-Bera and Sapiro-Wilk tests for normality and the Ljung-Box and LM-ARCH conditional heteroscedasticity tests for residuals. According to the statistical tests in the table, the residuals appear to be non-normal since the $p$-values of the two normality tests are less than $\alpha=0.05$, and they show no serial correlations in the series since the $p$-values of the Ljung-Box tests are greater than $\alpha=0.05$.

Table 6. The residual test results of the standard-GARCH $(1,1)$ model. It shows that the residuals are not normal and there is volatility clustering.

\begin{tabular}{ccc}
\hline Standardized Residuals (R) Tests & Statistic & $p$-Value \\
\hline Jarque-Bera Test on R & 6760.294 & 0.000 \\
Shapiro-Wilk Test on R & 0.824 & 0.000 \\
Ljung-Box Test on R Q(10) & 10.668 & 0.384 \\
Ljung-Box Test on R Q(15) & 12.946 & 0.606 \\
Ljung-Box Test on R Q(20) & 16.334 & 0.696 \\
Ljung-Box Test on R Squared Q(10) & 10.223 & 0.421 \\
Ljung-Box Test on R Squared Q(15) & 11.416 & 0.723 \\
Ljung-Box Test on R Squared Q(20) & 12.765 & 0.887 \\
LM-ARCH Test on R & 10.217 & 0.597 \\
\hline
\end{tabular}

We also consider nonlinear asymmetric GARCH to model LBTC, LSP, and LGD. Table 7 reports that there exists consistent volatility clustering since the $p$ values of $\beta_{1} s$ are all significant at $\alpha=0.05$, which is consistent with the results in Table 5 , and there is no volatility asymmetry in leverage effect in this period because all $p$ values of $\gamma_{1} s$ are not significant over each of the LBTC, LGD, LSP.

We built four different dynamic conditional correlation (DCC) models for LBTC and LSP and three different DCC models for LBTC and LGD. Figure 3 represents the DCC of four different models with DCC, NA-DCC, GC-DCC, and GCNA-DCC for log-returns of Bitcoin and S\&P 500. The patterns of the four models are almost similar to each other. However, the top two graphs for DCCs without Gaussian copulas are slightly different from the bottom two graphs for DCCs with Gaussian copulas for which 
NA-DCC using Gaussian copulas has relatively smaller values than those of using NA-DCC alone. In Figure 3, the highest positive DCC between LBTC and LSP was observed during the cryptocurrency crash in early 2018. In particular, we need to pay attention to that there exists a postive time-varying correlation between LBTC and LSP from March 2020 to September 2020 which is the COVID-19 pandemic period.

Table 7. Model fit of NA-GARCH $(1,1)$ where $\alpha_{0}, \alpha_{1}$, and $\beta_{1}$ are from Equation (2). Each of all $\beta_{1} \mathrm{~s}$ has significance indicating there exists consistent volatility clustering and all $\gamma_{1}$ s have no significance meaning there is no leverage effect (not asymmetric).

\begin{tabular}{|c|c|c|c|c|}
\hline \multicolumn{5}{|c|}{ NA-GARCH Model Fit with LBTC } \\
\hline & Estimate & S.E & $t$-Value & $p$-Value \\
\hline$\alpha_{0}$ & 0.000 & 0.000 & 0.298 & 0.765 \\
\hline$\alpha_{1}$ & 0.050 & 0.006 & 8.385 & 0.000 \\
\hline$\beta_{1}$ & 0.900 & 0.010 & 89.068 & 0.000 \\
\hline$\gamma_{1}$ & 0.050 & 0.068 & 0.737 & 0.461 \\
\hline t-distribution parameter & 4.000 & 0.215 & 18.605 & 0.000 \\
\hline \multicolumn{5}{|c|}{ NA-GARCH Model Fit with LSP } \\
\hline$\alpha_{0}$ & 0.000 & 0.000 & 0.073 & 0.942 \\
\hline$\alpha_{1}$ & 0.050 & 0.006 & 8.266 & 0.000 \\
\hline$\beta_{1}$ & 0.900 & 0.012 & 73.973 & 0.000 \\
\hline$\gamma_{1}$ & 0.051 & 0.059 & 0.861 & 0.389 \\
\hline t-distribution parameter & 4.000 & 0.208 & 19.215 & 0.000 \\
\hline \multicolumn{5}{|c|}{ NA-GARCH Model Fit with LGD } \\
\hline$\alpha_{0}$ & 0.000 & 0.000 & 0.923 & 0.356 \\
\hline$\alpha_{1}$ & 0.050 & 0.009 & 5.753 & 0.000 \\
\hline$\beta_{1}$ & 0.900 & 0.019 & 48.154 & 0.000 \\
\hline$\gamma_{1}$ & -0.003 & 0.089 & -0.037 & 0.971 \\
\hline t-distribution parameter & 4.000 & 0.268 & 14.921 & 0.000 \\
\hline
\end{tabular}

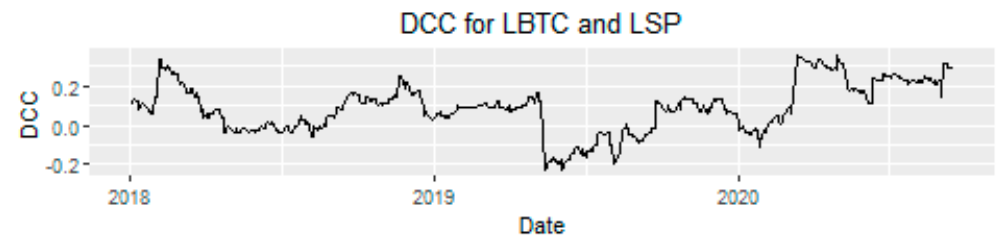

NA-DCC for LBTC and LSP

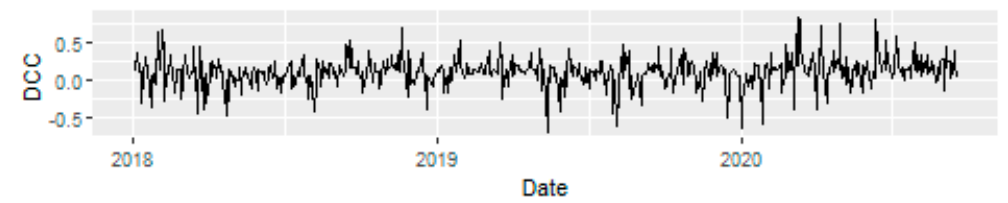

GC-DCC for LBTC and LSP

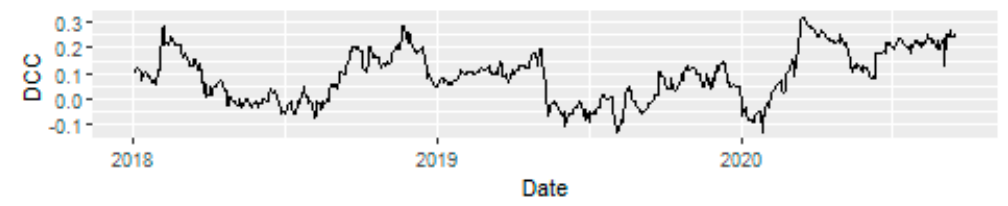

GCNA-DCC for LBTC and LSP

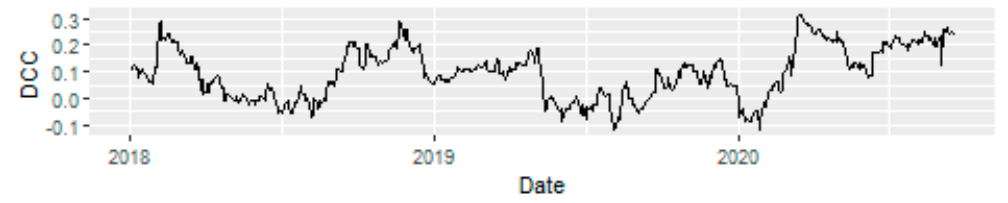

Figure 3. Dynamic conditional correlation between LBTC and LSP with GARCH-DCC (DCC), 
Nonlinear Asymmetric GARCH-DCC (NA-DCC), Gaussian copula-based GARCH-DCC (GC-DCC), and Gaussian copula-based Nonlinear Asymmetric GARCH-DCC (GCNA-DCC).

Figure 4 shows the plots describing the three models of DCC, GC-DCC, and GCNA-DCC for log-returns of Bitcoin and Gold. From the patterns of GC-DCC and GCNA-DCC in Figure 4, we also found that there exists a postive time-varying correlation between LBTC and LGD from March 2020 to September 2020 which is the COVID-19 pandemic period.
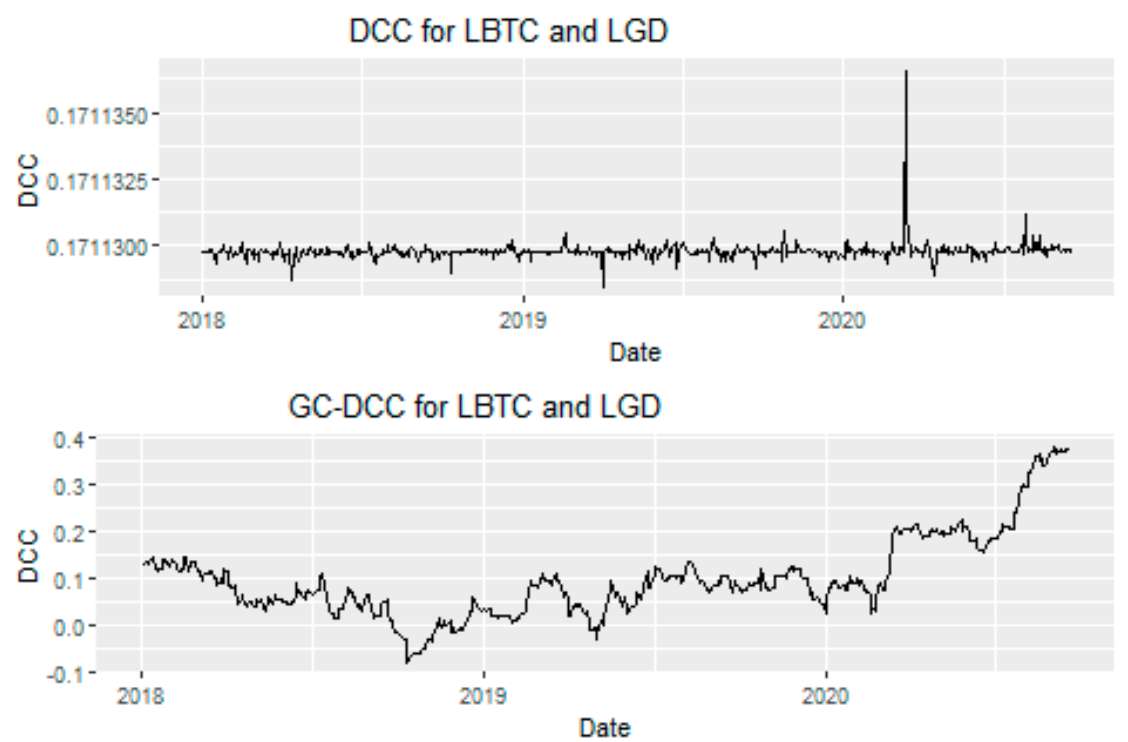

GCNA-DCC for LBTC and LGD

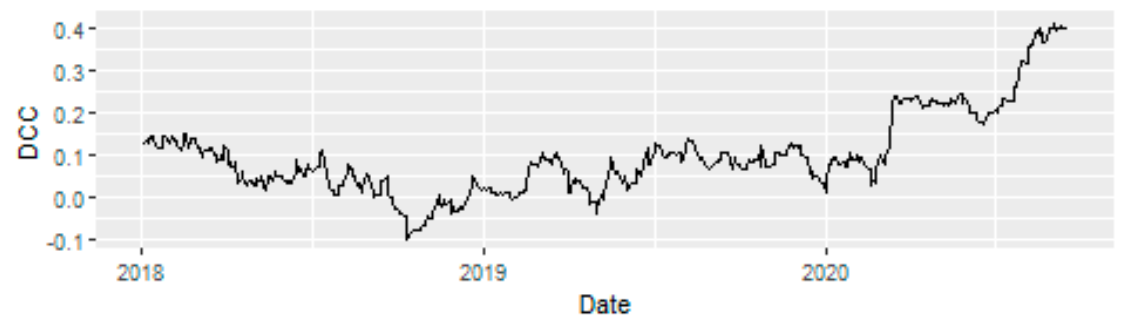

Figure 4. Dynamic conditional correlation between LBTC and LGD with GARCH-DCC (DCC), Gaussian copula-based GARCH-DCC (GC-DCC), and Gaussian copula-based Nonlinear Asymmetric GARCH-DCC (GCNA-DCC).

Log-likelihood is a measure of model fit. The higher the value, the better the fit. This is usually obtained from statistical output. For the pair of LBTC and LSP, the log-likelihood values of the DCC and NA-DCC models are smaller than the values of GC-DCC and GCNA-DCC in Table 8. Therefore, we can say that GC-DCC and GCNA-DCC are better models than DCC and NA-DCC to show relationship of Bitcoin with Gold and S\&P 500 in terms of log-likelihood. In addition, there is a computation difficulty to compute NA-DCC with LBTC and LGD. Therefore, we can conclude that our proposed method is a better statistical method to look at the relationship among financial assets compared with DCC and NA-DCC. In addition, the estimates of alpha and beta for GC-DCC and GCNA-DCC are statistically significant at the 5\% significance level but the estimates of alpha and beta for DCC and NA-DCC are not statistically significant at the $5 \%$ significance level. We can see that there is a computation difficulty to apply DCC and NA-DCC to high volatility financial data. The standard errors of the estimates from the DCC and NA-DCC models are much smaller than the standard errors from GC-DCC and GCNA-DCC. Especially, NA-DCC for Bitcoin and Gold cannot be computed from the "fGarch" R package [34] even though the log-likelihood value of DCC is larger than GC-DCC and GCNA-DCC. Based on these results, GC-DCC and GCNA-DCC are better models than DCC and NA-DCC. It is a 
strong motivation to apply the Gaussian copula DCC models for cryptocurrency to US stock and Gold market prices. We also investigate the relationship of the volatilities of cryptocurrency and US stock market with the GC-DCC or GCNA-DCC.

Table 8. The results of DCC with LBTC and LSP and with LBTC and LGD. Alpha and beta are the parameters for DCC, NA-DCC, GC-DCC and GCNA-DCC.

\begin{tabular}{|c|c|c|c|}
\hline DCC & & DCC Alpha & DCC Beta \\
\hline \multirow{3}{*}{ Bitcoin and S\&P 500} & Estimate & 0.025 & 0.953 \\
\hline & S.E & 0.038 & 0.067 \\
\hline & Log-likelihood & \multicolumn{2}{|c|}{3179.215} \\
\hline \multirow{3}{*}{ Bitcoin and Gold } & Estimate & 0.000 & 0.227 \\
\hline & S.E & 0.009 & 59,386 \\
\hline & Log-likelihood & \multicolumn{2}{|c|}{3326.434} \\
\hline \multirow[t]{2}{*}{ NA-DCC } & & NA-DCC Alpha & NA-DCC Beta \\
\hline & Estimate & 0.334 & 0.188 \\
\hline \multirow[t]{2}{*}{ Bitcoin and S\&P 500} & S.E & 0.109 & 0.591 \\
\hline & Log-likelihood & \multicolumn{2}{|c|}{2754.319} \\
\hline \multirow{3}{*}{ Bitcoin and Gold } & Estimate & NA & NA \\
\hline & S.E & NA & NA \\
\hline & Log-likelihood & \multicolumn{2}{|c|}{ NA } \\
\hline GC-DCC & & GC-DCC Alpha & GC-DCC Beta \\
\hline \multirow{3}{*}{ Bitcoin and S\&P 500} & Estimate & 0.069 & 0.910 \\
\hline & S.E & 0.013 & 0.019 \\
\hline & Log-likelihood/AIC & \multicolumn{2}{|c|}{$3286.216 /-10.0129$} \\
\hline \multirow{3}{*}{ Bitcoin and Gold } & Estimate & 0.069 & 0.910 \\
\hline & S.E & 0.013 & 0.019 \\
\hline & Log-likelihood/AIC & \multicolumn{2}{|c|}{$3437.459 /-10.475$} \\
\hline \multirow[t]{2}{*}{ GCNA-DCC } & & GCNA-DCC Alpha & GCNA-DCC Beta \\
\hline & Estimate & 0.068 & 0.899 \\
\hline \multirow[t]{2}{*}{ Bitcoin and S\&P 500} & S.E & 0.015 & 0.063 \\
\hline & Log-likelihood/AIC & \multicolumn{2}{|c|}{$3293.257 /-10.028$} \\
\hline \multirow{3}{*}{ Bitcoin and Gold } & Estimate & 0.068 & 0.899 \\
\hline & S.E & 0.015 & 0.063 \\
\hline & Log-likelihood/AIC & \multicolumn{2}{|c|}{$2143.902 /-10.028$} \\
\hline
\end{tabular}

NA means no computational result because of an optimization error from the " $f G a r c h " ~ R$ package.

We have two hypotheses from this research. The first hypothesis is that we want to test the alternative hypothesis that Bitcoin is statistically significant by log-returns of S\&P 500 and Gold price in terms of log-return. The second hypothesis is that we also test another alternative hypothesis that the current volatility of log-returns of Bitcoin can be statistically significant with the current and lagged volatilities of the other assets (S\&P 500 and Gold price).

To perform the first alternative hypothesis that Bitcoin is statistically significant by log-returns of S\&P 500 and Gold price in terms of log-return, we consider building an optimal Autoregressive Moving Average (ARMA) model based on AIC criteria among four different combinations of $\mathrm{p}$ and q: $(0,0),(0,1),(1,0)$, and $(1,1)$. Table 9 shows the result of the selection of $p$ and $q$ for the ARMA model. The ARMA $(0,0)$ turned out to be the best model with a minimum AIC value and Table 9 shows the result of the GCMR model fit of LBTC with LSP and LGD with error dependence structure of ARMA $(0,0)$. The reason we employ GCMR for the modeling is that GCMR has a Sigma dispersion parameter which accounts for heteroscedasticity of error. The GCMR model is more flexible to model the data which do not follow normality or heteroscedasticity of errors. Table 9 shows that there exists a statistical significance between LSP and LGD to LBTC in terms of price. And the Sigma dispersion parameter is statistically significant at the $5 \%$ significance level. 
Table 9. Selection of $\mathrm{p}$ and $\mathrm{q}$ for ARMA based on AIC of 4 cases of $(0,0),(0,1),(1,0)$, and $(1,1)$. ARMA $(0,0)$ is selected based on AIC criteria. CMR model fit of LBTC with LSP and LGD with error dependence structure ARMA $(0,0)$.

\begin{tabular}{ccccc}
\hline Model & \multicolumn{4}{c}{ LBTC $=$ Intercept $+\alpha_{1} \times$ LSP $+\beta_{1} \times$ LGD } \\
\hline ARMA $(\mathrm{p}, \mathrm{q})$ & ARMA $(0,0)$ & ARMA $(0,1)$ & ARMA $(1,0)$ & ARMA $(1,1)$ \\
AIC & -2140.6 & -2139.7 & -2139.8 & -2138.5 \\
ARMA $(0,0)$ & & LBTC $=$ Intercept $+\alpha_{1} \times$ LSP $+\beta_{1} \times$ LGD & \\
& Estimate & S.E & Z-value & $p$-value \\
Intercept & -0.001 & 0.002 & -0.653 & 0.514 \\
LSP & 0.645 & 0.124 & 5.182 & 0.000 \\
LGD & 0.803 & 0.208 & 3.860 & 0.000 \\
Sigma & 0.047 & 0.001 & 36.163 & 0.000 \\
\hline
\end{tabular}

With volatilities by both standard-GARCH $(1,1)$ and nonlinear asymmetric GARCH $(1,1)$, we compare the values of both AIC and Log Likelihood for LBTC Volatility $(\mathrm{t})=$ Intercept $+\alpha_{1} \times$ LSP Volatility $(\mathrm{t})+\alpha_{2} \times$ LGD Volatility $(\mathrm{t})+\alpha_{3} \times$ LSP Volatility $(\mathrm{t}-1)+\alpha_{4} \times$ LGD Volatility $(\mathrm{t}-1)$ where $\mathrm{t}-1$ is one day before and $\mathrm{t}=2, \ldots, 401$ in Tables 10 and 11 .

Table 10. With standard-GARCH $(1,1)$ volatilities, GCMR model fit of LBTC volatility with LSP volatility $(\mathrm{t})$, LGD volatility $(\mathrm{t})$, volatility $(\mathrm{t}-1)$ and LGD volatility $(\mathrm{t}-1)$ with error dependence structure ARMA $(0,0)$.

\begin{tabular}{|c|c|c|c|c|}
\hline \multirow[t]{2}{*}{ Model } & \multicolumn{4}{|c|}{$\begin{array}{c}\text { LBTC Volatility }(\mathrm{t})=\text { Intercept }+\alpha_{1} \times \text { LSP Volatility }(\mathrm{t})+\alpha_{2} \times \text { LGD } \\
\text { Volatility }(\mathrm{t})+\alpha_{3} \times \text { LSP Volatility }(\mathrm{t}-1)+\alpha_{4} \times \text { LGD Volatility }(\mathrm{t}-1)\end{array}$} \\
\hline & Estimate & S.E. & Z-value & $p$-value \\
\hline Intercept & -6.698 & 0.262 & -25.570 & 0.000 \\
\hline LSP Volatility (t) & 1341.294 & 0.015 & 90993.019 & 0.000 \\
\hline LGD Volatility (t) & 17.942 & 3.303 & 5.431 & 0.000 \\
\hline LSP Volatility (t-1) & 6350.534 & 0.008 & 771671.098 & 0.013 \\
\hline LGD Volatility (t-1) & -11.556 & 3.662 & -3.156 & 0.001 \\
\hline Shape & 0.800 & 0.209 & 3.818 & 0.000 \\
\hline Log Likelihood & \multirow{2}{*}{\multicolumn{4}{|c|}{$\begin{array}{l}-5799.7 \\
-11583\end{array}$}} \\
\hline AIC & & & & \\
\hline
\end{tabular}

Table 11. With nonlinear asymmetric GARCH $(1,1)$ volatilities, GCMR model fit of LBTC volatility with LSP volatility $(\mathrm{t})$, LGD volatility $(\mathrm{t})$, volatility $(\mathrm{t}-1)$ and LGD volatility $(\mathrm{t}-1)$ with error dependence structure ARMA $(0,0)$.

\begin{tabular}{|c|c|c|c|c|}
\hline \multirow[t]{2}{*}{ Model } & \multicolumn{4}{|c|}{$\begin{array}{l}\text { LBTC Volatility }(\mathrm{t})=\text { Intercept }+\alpha_{1} \times \text { LSP Volatility }(\mathrm{t})+\alpha_{2} \times \text { LGD } \\
\text { Volatility }(\mathrm{t})+\alpha_{1} \times \text { LSP Volatility }(\mathrm{t}-1)+\alpha_{2} \times \text { LGD Volatility }(\mathrm{t}-1)\end{array}$} \\
\hline & Estimate & S.E. & Z-value & $p$-value \\
\hline Intercept & -6.085 & 0.109 & -55.757 & 0.000 \\
\hline LSP Volatility (t) & 24.313 & 0.032 & 760.170 & 0.000 \\
\hline LGD Volatility (t) & 5.092 & 0.583 & 8.739 & 0.000 \\
\hline LSP Volatility (t-1) & 89.167 & 0.021 & 4291.506 & 0.000 \\
\hline LGD Volatility $(\mathrm{t}-1)$ & -2.085 & 0.636 & -3.279 & 0.001 \\
\hline Sigma & 1.388 & 0.091 & 15.295 & 0.000 \\
\hline Log Likelihood & \multirow{2}{*}{\multicolumn{4}{|c|}{$\begin{array}{l}-3837.7 \\
-7661.5\end{array}$}} \\
\hline AIC & & & & \\
\hline
\end{tabular}

The GCMR model fit of LBTC volatility $(\mathrm{t})$ with LSP volatility $(\mathrm{t})$, LGD volatility $(\mathrm{t})$, volatility $(\mathrm{t}-1)$, and LGD volatility $(\mathrm{t}-1)$ with standard-GARCH $(1,1)$ volatilities and error dependence structure ARMA $(0,0)$ is better than the GCMR model fit of LBTC volatility $(t)$ with LSP volatility $(t)$, LGD volatility $(t)$, volatility $(\mathrm{t}-1)$, and LGD volatility $(\mathrm{t}-1)$ with nonlinear asymmetric $\mathrm{GARCH}(1,1)$ volatilities and error dependence structure ARMA $(0,0)$. 
We chose the statistical output from Table 10 so that LSP volatility ( $\mathrm{t}$ ), LGD volatility (t) and LSP volatility ( $\mathrm{t}-1)$ are statistically significant, and they have a positive statistical effect to LBTC volatility $(t)$, but LGD volatility ( $t-1)$, one day before volatilities, has a statistically significant negative effect to LBTC volatility $(\mathrm{t})$ at the $5 \%$ significance level. The Sigma dispersion parameter is also statistically significant at the 5\% significance level in both Tables 10 and 11.

The following statistical output is another interesting result in our paper. We want to see the relationship of the Gaussian copula time-varying correlation (GC-DCC or GCNA-DCC) with the volatilities of LBTC and LSP. With volatilities by both standard-GARCH $(1,1)$ and nonlinear asymmetric GARCH $(1,1)$ with an error dependence structure of ARMA $(1,0)$, we also compared the log-likelihood of GC-DCC $=$ Intercept $+\alpha_{1} \times$ LBTC Volatility $+\beta_{1} \times$ LSP Volatility with GCNA-DCC $=$ Intercept + $\alpha_{1} \times$ LBTC Volatility $+\beta_{1} \times$ LSP Volatility in Tables 12 and 13 .

Table 12. Gaussian Copula Marginal Regression (GCMR) with standard-GARCH $(1,1)$ volatilities. Selection of $p$ and $q$ for ARMA based on AIC of 4 cases of $(0,0),(0,1),(1,0)$, and $(1,1)$. ARMA $(0,0)$ is selected based on AIC criteria. GCMR Model fit of GC-DCC with LBTC Volatility, and LSP Volatility of Error dependence structure ARMA $(1,1)$.

\begin{tabular}{ccccc}
\hline Model & \multicolumn{4}{c}{ GC-DCC $=$ Intercept $+\alpha_{1} \times$ LBTC Volatility $+\boldsymbol{\beta}_{1} \times$ LSP Volatility } \\
\hline ARMA $(\mathrm{p}, \mathrm{q})$ & ARMA $(0,0)$ & ARMA $(0,1)$ & ARMA $(1,0)$ & ARMA $(1,1)$ \\
AIC & -1312.2 & -1960.7 & NA & -3014.6 \\
ARMA (1, 1) & GC-DCC & Intercept $+\alpha_{1} \times$ LBTC & Volatility $+\beta_{1} \times$ LSP Volatility \\
& Estimate & S.E & z-value & $P$-value \\
Intercept & 0.068 & 0.026 & 2.580 & 0.010 \\
LBTC Volatility & 1.912 & 4.698 & 0.407 & 0.684 \\
LSP Volatility & 371.367 & 0.269 & 1379.714 & 0.000 \\
Sigma & 0.089 & 0.013 & 6.625 & 0.000 \\
Log-likelihood & & \multicolumn{4}{c}{-1513.3} \\
\hline
\end{tabular}

Table 13. Gaussian Copula Marginal Regression with nonlinear asymmetric GARCH $(1,1)$ volatilities. Selection of $p$ and $q$ for ARMA based on AIC of 4 cases of $(0,0),(0,1),(1,0)$, and $(1,1)$. ARMA $(1$, 0 ) is selected based on AIC criteria. GCMR Model fit of GCNA-DCC with LBTC Volatility, and LSP Volatility of Error dependence structure $\operatorname{ARMA}(1,1)$.

\begin{tabular}{ccccc}
\hline Model & \multicolumn{4}{c}{ GCNA-DCC $=$ Intercept $+\alpha_{1} \times$ LBTC Volatility $+\boldsymbol{\beta}_{1} \times$ LSP Volatility } \\
\hline ARMA $(\mathrm{p}, \mathrm{q})$ & ARMA $(0,0)$ & ARMA $(0,1)$ & ARMA $(1,0)$ & ARMA $(1,1)$ \\
AIC & -678.24 & -1994.3 & NA & -3070.4 \\
ARMA(1, 1) & GCNA-DCC & Intercept $+\alpha_{1} \times$ LBTC & Volatility $+\beta_{1} \times$ LSP Volatility \\
& Estimate & S.E & Z-value & $P$-value \\
Intercept & 0.087 & 0.027 & 3.223 & 0.001 \\
LBTC Volatility & 16.698 & 4.572 & 3.652 & 0.000 \\
LSP Volatility & 131.869 & 0.264 & 499.807 & 0.000 \\
Sigma & 0.088 & 0.014 & 6.377 & 0.000 \\
Log-likelihood & & \multicolumn{4}{c}{-1541.2} \\
\hline
\end{tabular}

From the relationship among time-varying conditional correlation with LBTC volatility, and LSP volatility by the Gaussian Copula Marginal Regression (GCMR) Model in Tables 12 and 13, we find that there exists a statistically significant and positive effect to time-varying conditional correlation by the volatility of LBTC and the volatility of LSP.

\section{Conclusions}

We applied the copula-based GARCH-DCC models to the financial assets, Bitcoin, Gold, and S\&P 500. We showed that the proposed method for the relationships among time-varying conditional correlation with Bitcoin volatility, and S\&P 500 can overcome the difficulty which cannot be computed by the GARCH-DCC models. Our empirical study showed the time-varying relationship between 
the cryptocurrency market and the US stock market or the gold market price. Recent data showed that there was a positive time-varying relationship between these two markets since the COVID-19 occurrence. Our Gaussian copula marginal regression modeling the volatility of the most popular cryptocurrency, Bitcoin, with Gold price and US stock market price has more performance compared to competitors such as DCC and NA-DCC to show that a volatility relationship exists among the three market prices with the current day and one-day lagged prices. Our findings provide important implications for both investors and policymakers. In our future study, we will apply state-space modeling for the most popular cryptocurrency with the Gold price and US stock market to see a time-varying relationship in terms of a time-varying intercept and slope. The limitation of this research is that our proposed copula DCC methodology to the high volatility finance assets is not multivariate data analysis but pairwise data analysis. In order to overcome this limitation, our future study will be based on multivariable time series data by using vine copula based multivariate time varying correlation analysis so that we will be able to look at the multivariate time varying correlation behavior among several financial assets simultaneously.

Author Contributions: Formal analysis and investigation, J.-M.K. and S.K.; writing—original draft preparation, S.K., J.-M.K. and S.-T.K.; supervision and reviewing, S.K. All authors have read and agreed to the published version of the manuscript.

Funding: This research was funded by Dong-A University, South Korea.

Acknowledgments: We would like to thank the editor and reviewers for their insightful comments and helpful suggestions.

Conflicts of Interest: The authors declare no conflict of interest.

\section{References}

1. Nakamoto, S. Bitcoin: A Peer-to-Peer Electronic Cash System. Available online: https://bitcoin.org/bitcoin.pdf (accessed on 3 May 2018).

2. Katsiampa, P. An empirical investigation of volatility dynamics in the cryptocurrency market. Res. Int. Bus. Finance 2019, 50, 322-335. [CrossRef]

3. Hyun, S.; Lee, J.M.; Kim, J.; Jun, C. What coins lead in the cryptocurrency market? Using Copula and Neural Network Models. J. Risk Financ. Manag. 2019, 12, 132. [CrossRef]

4. Kim, J.-M.; Jun, C.; Lee, J. Forecasting the volatility of the cryptocurrency market using GARCH and Stochastic Volatility. Econ. Model. 2019. under review.

5. Klein, T.; Thu, H.P.; Walther, T. Bitcoin is not the New Gold-A comparison of volatility, correlation, and portfolio performance. Int. Rev. Financ. Anal. 2018, 59, 105-116. [CrossRef]

6. Baba, Y.; Engle, R.F.; Kraft, D.F.; Kroner, K.F. Multivariate simultaneous generalized ARCH. Econ. Theory 1995, 11, 122-150.

7. Aslanidisa, N.; Barivierab, A.F.; Martínez-Ibañeza, O. An analysis of cryptocurrencies conditional cross correlations. Financ. Res. Lett. 2019, 31, 130-137. [CrossRef]

8. Ghosh, I.; Sanyal, M.K.; Jana, R.K. Co-movement and Dynamic Correlation of Financial and Energy Markets: An Integrated Framework of Nonlinear Dynamics, Wavelet Analysis and DCC-GARCH. Comput. Econ. 2020. [CrossRef]

9. Maraqa, B.; Bein, M. Dynamic Interrelationship and Volatility Spillover among Sustainability Stock Markets, Major European Conventional Indices, and International Crude Oil. Sustainability 2020, 12, 3908. [CrossRef]

10. Chen, Y.; Qu, F. Leverage effect and dynamics correlation between international crude oil and China's precious metals. Phys. A Stat. Mech. Appl. 2019, 534. [CrossRef]

11. Lee, N.; Kim, J.-M. Dynamic functional connectivity analysis of functional MRI based on copula time-varying correlation. J. Neurosci. Methods 2019, 323, 32-47. [CrossRef]

12. Guesmi, K.; Saadi, S.; Abid, I.; Ftiti, Z. Portfolio diversification with virtual currency: Evidence from bitcoin. Int. Rev. Financ. Anal. 2019, 63, 431-437. [CrossRef]

13. Denkowska, A.; Wanat, S. A Tail Dependence-Based MST and Their Topological Indicators in Modeling Systemic Risk in the European Insurance Sector. Risks 2020, 8, 39. [CrossRef] 
14. Chen, H.; Liu, Z.; Zhang, Y.; Wu, Y. The Linkages of Carbon Spot-Futures: Evidence from EU-ETS in the Third Phase. Sustainability 2020, 12, 2517. [CrossRef]

15. Lee, N.; Kim, J.-M. Dynamic functional connectivity analysis based on time-varying partial correlation with a copula-DCC-GARCH model. Neurosci. Res. 2020. [CrossRef]

16. John, M.; Wu, Y.; Narayan, M.; John, A.; Ikuta, T.; Ferbinteanu, J. Estimation of Dynamic Bivariate Correlation Using a Weighted Graph Algorithm. Entropy 2020, 22, 617. [CrossRef]

17. Amrouk, E.M.; Grosche, S.C.; Heckelei, T. Interdependence between cash crop and staple food international prices across periods of varying financial market stress. Appl. Econ. 2020, 52. [CrossRef]

18. Kim, J.M.; Jun, S. Graphical causal inference and copula regression model for apple keywords by text mining. Adv. Eng. Inform. 2015, 29, 918-929. [CrossRef]

19. Masarotto, G.; Varin, C. Gaussian Copula Marginal Regression. Electron. J. Stat. 2012, 6, 1517-1549. [CrossRef]

20. Hentschel, L. All in the Family Nesting Symmetric and Asymmetric GARCH Models. J. Financ. Econ. 1995, 39, 71-104. [CrossRef]

21. Engle, R.F.; Ng, V.K. Measuring and Testing the Impact of News on Volatility. J. Financ. 1993, 48, $1749-1778$. [CrossRef]

22. Tse, Y.K.; Tsui, A.K.C. A multivariate generalized autoregressive conditional heteroscedasticity model with time-varying correlations. J. Bus. Econ. Stat. 2002, 20, 351-362. [CrossRef]

23. Bollerslev, T. Modeling the coherence in short-run nominal exchange rates: A multivariate generalized ARCH model. Rev. Econ. Stat. 1990, 72, 498-505. [CrossRef]

24. Engle, R. Dynamic conditional correlation: A simple class of multivariate generalized autoregressive conditional heteroskedasticity models. J. Bus. Econ. Stat. 2002, 20, 339-350. [CrossRef]

25. Engle, R.F.; Sheppard, K. Theoretical and Empirical Properties of Dynamic Conditional Correlation Multivariate GARCH; Working Paper 8554; National Bureau of Economic Research: Cambridge, MA, USA, 2011. [CrossRef]

26. Sklar, M. Fonctions de Répartition À N Dimensions et Leurs Marges; Université Paris: Paris, France, 1959.

27. Joe, H. Multivariate Models and Dependence Concepts; Chapman \& Hall: London, UK, 1997.

28. Genest, C.; Ghoudi, K.; Rivest, L. A semiparametric estimation procedure of dependence parameters in multivariate families of distributions. Biometrica 1995, 82, 543-552. [CrossRef]

29. Song, P. Multivariate Dispersion Models Generated from Gaussian Copula. Scand. J. Stat. 2000, 27, 305-320. [CrossRef]

30. Crypto-Defi Wallet-CoinMarketCap. Available online: https://coinmarketcap.com/coins/ (accessed on 2 April 2018).

31. University of British Columbia, Sauder School of Business. Pacific Exchange Rate Service. Available online: http://fx.sauder.ubc.ca/data.html (accessed on 5 January 2020).

32. Yahoo Finance. Available online: https://finance.yahoo.com/ (accessed on 5 January 2020).

33. Dumitrescu, E.I.; Hurlin, C. Testing for Granger non-causality in heterogeneous panels. Econ. Model. 2012, 29, 1450-1460. [CrossRef]

34. Bollerslev, T. Generalized Autoregressive Conditional Heteroscedasticity. J. Econ. 1986, 31, 307-327. [CrossRef]

Publisher's Note: MDPI stays neutral with regard to jurisdictional claims in published maps and institutional affiliations.

(C) 2020 by the authors. Licensee MDPI, Basel, Switzerland. This article is an open access article distributed under the terms and conditions of the Creative Commons Attribution (CC BY) license (http://creativecommons.org/licenses/by/4.0/). 